\title{
Reduced Fading of Visual Afterimages after Transcranial Magnetic Stimulation over Early Visual Cortex
}

Citation for published version (APA):

Engelen, T., Rademaker, R. L., \& Sack, A. T. (2019). Reduced Fading of Visual Afterimages after Transcranial Magnetic Stimulation over Early Visual Cortex. Journal of Cognitive Neuroscience, 31(9), 1368-1379. https://doi.org/10.1162/jocn_a_01415

Document status and date:

Published: 01/09/2019

DOI:

10.1162/jocn_a_01415

Document Version:

Publisher's PDF, also known as Version of record

Document license:

Taverne

Please check the document version of this publication:

- A submitted manuscript is the version of the article upon submission and before peer-review. There can be important differences between the submitted version and the official published version of record.

People interested in the research are advised to contact the author for the final version of the publication, or visit the DOI to the publisher's website.

- The final author version and the galley proof are versions of the publication after peer review.

- The final published version features the final layout of the paper including the volume, issue and page numbers.

Link to publication

\footnotetext{
General rights rights.

- You may freely distribute the URL identifying the publication in the public portal. please follow below link for the End User Agreement:

www.umlib.nl/taverne-license

Take down policy

If you believe that this document breaches copyright please contact us at:

repository@maastrichtuniversity.nl

providing details and we will investigate your claim.
}

Copyright and moral rights for the publications made accessible in the public portal are retained by the authors and/or other copyright owners and it is a condition of accessing publications that users recognise and abide by the legal requirements associated with these

- Users may download and print one copy of any publication from the public portal for the purpose of private study or research.

- You may not further distribute the material or use it for any profit-making activity or commercial gain

If the publication is distributed under the terms of Article $25 \mathrm{fa}$ of the Dutch Copyright Act, indicated by the "Taverne" license above, 


\title{
Reduced Fading of Visual Afterimages after Transcranial Magnetic Stimulation over Early Visual Cortex
}

\author{
Tahnée Engelen $^{1,2 *}$, Rosanne L. Rademaker ${ }^{3,4 *}$, and Alexander T. Sack
}

\begin{abstract}
In the complete absence of small transients in visual inputs (e.g., by experimentally stabilizing an image on the retina or in everyday life during intent staring), information perceived by the eyes will fade from the perceptual experience. Although the mechanisms of visual fading remain poorly understood, one possibility is that higher level brain regions actively suppress the stable visual signals via targeted feedback onto early visual cortex (EVC). Here, we used positive afterimages and multisensory conflict to induce gestalt-like fading of participants' own hands. In two separate experiments, participants rated the perceived quality of their hands both before and after transcranial magnetic
\end{abstract}

\section{INTRODUCTION}

Our perception of the world is the result of smooth integration of a multitude of senses. This integration enables us to move through, interpret, and interact with our environment with ease. Of particular importance is the integration of visual and proprioceptive information, as integration between these two modalities helps guide our actions. In everyday life, visual and proprioceptive modalities will generally be in congruence with one another. That is, when reaching for an object, you will both see your hand reaching toward the object, and receive proprioceptive feedback on the trajectory of your hand. Despite the normally observed congruence between vision and proprioception, circumstances can be created during which information conveyed by these two modalities is in conflict. Studying such circumstances can give valuable information on how sensory conflict is resolved and how sensory cortices have the ability to exert influence over one another.

One way to create a sensory conflict between vision and proprioception is through the use of positive afterimages (Davies, 1973). Positive afterimages can be elicited by discharging a brief flash of light after a prolonged period of dark adaptation. In the total darkness after the

\footnotetext{
${ }^{1}$ Maastricht University, ${ }^{2}$ École Normale Supérieure-PSL University, ${ }^{3}$ University of California, San Diego, ${ }^{4}$ Radboud University

*Denotes equal contributions.
}

stimulation (TMS) was applied over EVC. In a first experiment, triple-pulse TMS was able to make a faded hand appear less faded after the pulses were applied, compared with placebo pulses. A second experiment demonstrated that this was because triplepulse TMS slowed down fading of the removed hand that otherwise occurs naturally over time. Interestingly, TMS similarly affected the left and right hands, despite being applied only over the right EVC. Together, our results suggest that TMS over EVC attenuates the effects of visual fading in positive afterimages, and it might do so by crossing transcollosal connections or via multimodal integration sites in which both hands are represented. flash, a grayscale visual afterimage develops of everything that was perceived during the flash. The experience can be described as a dim light turning on, with lights and darks having the correct sign (i.e., contrast is not inverted). A subsequent conflict between vision and proprioception can be created by having people move parts of their body. For example, when an afterimage is elicited while one is viewing one's own hand, and subsequently that hand is moved out of the afterimage, these two modalities will send incongruent messages to the brain (i.e., proprioception indicates the hand is removed, whereas the retinal input indicates the hand is still in view). As a result of the relocation of the hand, the image of the hand will appear to fade or crumble, whereas the rest of the afterimage stays intact (Davies, 1973; Gregory, Wallace, \& Campbell, 1959). In this case, proprioception has a catastrophic impact on visual perception as conflicting information is encoded from the two senses. Note that this resolution to the sensory conflict is somewhat unique, as in most cases a conflict between vision and proprioception is resolved in favor of the visual sense (Hay, Pick, \& Ikeda, 1965), presumably due to its high spatial acuity and reliable signals.

As the above example demonstrates, positive afterimages provide a powerful means to impact visual perceptions via manipulations of proprioception. In another example, when a person moves their hand closer to or further from their eyes after a positive afterimage of the hand has been created, such movements can induce a perceived change in size: A hand will appear to 
shrink or grow, depending on the direction of the movement (i.e., toward or away from the eyes, respectively; Carey \& Allan, 1996). Both fading and size scaling of a hand in an afterimage occur irrespective of whether the movement is active or passive, suggesting that afferent proprioceptive information is sufficient to influence the visual percept (Hogendoorn, Kammers, Carlson, \& Verstraten, 2009; Bross, 2000).

Neural correlates of the marked influence that proprioception can have on our visual experience during positive afterimages have not been extensively studied. Luckily, there are good indications of the underlying mechanisms within the broader literature on visual fading. If you have ever stared steadily enough at a paint chip on a wall or a crack in the ceiling, you may have noticed the peripheral world slowly fading into oblivion. This perceptual experience is known as the Troxler effect (Troxler, 1804), and is just one of many examples of visual fading (Billock \& Tsou, 2004; Riggs, Ratliff, Cornsweet, \& Cornsweet, 1953; Ditchburn \& Ginsborg, 1952; Darwin, 1794). It is generally understood that small transients in the visual input, such as those induced via microsaccades, are critical to establish a conscious visual percept, whereas images that are stable on the retina will result in the visual world fading away (Martinez-Conde, Otero-Millan, \& Macknik, 2013; Martinez-Conde, Macknik, \& Hubel, 2004). This relates directly to positive afterimages, which are believed to stem from retinal rod activity and therefore provide an elegant means to achieve retinal stabilization.

It is widely recognized that visual percepts are shaped by more than solely the input originating from the retina. This is evident from the previously described proprioceptive influences observed in positive afterimages, which drastically alter the visual percept. During perceptual fading, an image is sensed at the level of the retina while that image is not being perceived (Martinez-Conde et al., 2004, 2013; Billock \& Tsou, 2004). By contrast, during perceptual filling-in, information that is not directly sensed by the eyes is conciously perceived (Komatsu, 2006; Shimojo, Kamitani, \& Nishida, 2001). In all these examples, the subjective percept, and not retinal input, seems to determine responses at the earliest levels of visual processing. In perceptual filling-in, fMRI activity in primary visual area V1 tracked the subjectively perceived filled-in surface between two moving gratings (Meng, Remus, \& Tong, 2005) and an illusory colored surface (Sasaki \& Watanabe, 2004), rather than the objectively presented blank gap. Similarly, modulations of retinotopic activity in V1 have been demonstrated under conditions of constant retinal inputs: Retinotopic activity tracked the perceived size changes of retinally identical afterimages (Sperandio, Chouinard, \& Goodale, 2012), and objects that appeared to occupy more space in the visual field (but had the same angular size) also activated a greater portion of V1 than objects that appeared to occupy less space (Murray, Boyaci, \& Kersten, 2006). Finally, lower levels of visuocortical activity have been observed when a visual image (a monochromatic disk) faded into the background, com- pared with when it was consciously perceived (Mendola, Conner, Sharma, Bahekar, \& Lemieux, 2006). Thus, changes in conscious visual percepts are accompanied by changes at the earliest cortical levels of visual processing (i.e., in early visual cortex [EVC]).

It is common for perceptual fading to occur in meaningful chunks, following Gestalt-like principles (Billock \& Tsou, 2004). For example, in the positive afterimage only the hand will fade once moved out of sight, whereas the rest of the image stays intact. This implies the involvement of higher level areas able to process information at a more semantic level. Indeed, the neural correlates of the fading monochromatic disk extended beyond V1 and were also observed in higher level areas, including parietal cortex (Mendola et al., 2006). Support for a causal link between parietal cortex and conscious visual percepts comes from a study showing that transcranial magnetic stimulation (TMS) over intraparietal sulcus was able to elicit visual fading of a peripherally presented target. Presumably, TMS led to visual fading because it degraded the quality of attentional feedback signals to EVC (Kanai, Muggleton, \& Walsh, 2008).

Given the interplay between both higher and lower level brain regions, the question arises which neural mechanisms may underlie the perceptual fading in positive afterimages caused by bodily movements. Because changes in early sensory cortex generally track perception, here we hypothesize that dynamics at the level of EVC are responsible for this form of visual fading. Fading of the hand might ensue when ongoing but stable visual inputs are actively filtered out of the conscious experience - a process instantiated by proprioceptive signals. Targeted top-down inputs from multimodal areas onto EVC might be a means to impose the early-level changes required for the conscious experience of gestaltlike fading in positive afterimages (Bolognini \& Maravita, 2007; Macaluso, Frith, \& Driver, 2000). Specifically, fading of the hand could be due to the active inhibition of the bottom-up retinal signals associated with viewing that hand. Although this type of fading is necessarily instantiated by signals originating from outside EVC, it might be sustained via continued feedback from higher level areas or via local mechanisms at the level of EVC. In either case, we hypothesize that active inhibition at the level of EVC is responsible for the perceptual experience of a hand fading in an afterimage. Speculatively, active inhibition might more broadly serve as the mechanism responsible for perceptual fading of stabilized images: In the absence of visual transients, information might be deemed irrelevant by the visual system and hence systematically filtered out of the perceptual experience.

Here, we tested this hypothesis by combining fading in positive afterimages with online TMS over the right EVC. If perceived gestalt-like fading of a hand is indeed the result of targeted inhibition at the level of EVC, disrupting this inhibition by means of TMS should weaken the 
extent to which the hand is perceived to fade (i.e., lead to a temporary "recovery" of the faded hand). In two separate experiments, participants viewed afterimages of both their hands held out in front of them. In Experiment 1 , one of the hands was removed, and participants rated the relative clarity of their two hands immediately before and immediately after a TMS manipulation over the right EVC. Manipulations consisted of a single TMS pulse, a triple TMS pulse, or a single placebo TMS pulse. In Experiment 2, only triple pulses (placebo and real TMS) were administered, and participants rated both hands individually to disentangle the effects of TMS on hands ipsilateral and contralateral to the targeted right hemisphere. In both experiments, we demonstrated that the gestalt-like fading of the removed hand was systematically reduced after administering TMS over EVC. This implies that TMS can provide temporary relief from the factors responsible for perceptual fading and suggests that active inhibitory mechanisms impacting visual processing at the level of EVC are likely at play during perceptual fading.

\section{METHODS}

\section{Participants}

A total of 29 participants were recruited from Maastricht University to partake in an initial screening session, where we tested if participants met the required criteria to be included in the main experiment (see Procedure section for more details). Based on this screening, we excluded nine people from participation on the grounds that they were unable to perceive phosphenes $(n=2)$, unable to perceive phosphenes at the desired visual field location $(n=1)$, unable to experience an afterimage long enough to give the ratings within the time the image was perceived $(n=2)$, or unable to experience fading in the afterimage in response to bodily movement $(n=4)$.
Thus, of the 29 originally recruited people, only 20 advanced to participate in the main experimental procedures. Participants in Experiment 1 were 10 healthy volunteers with a mean age of 25.8 years $(S E=3.3$; eight women). Ten more participants took part in Experiment 2, of whom two had previously participated in Experiment 1 (mean age $=25.5$ years, $S D=2.8$; six women). All participants were right-handed, had normal or correctedto-normal vision, were naïve to the purpose of the experiment, and received monetary reimbursement for their time (except for author R. R. in Experiment 2). Before the start of the experimental proceedings, participants provided written informed consent and were screened for TMS safety based on published safety guidelines (Rossi, Hallett, Rossini, Pascual-Leone, \& The Safety of TMS Concensus Group, 2009). This study was approved by the standing ethical committee of the Psychology and Neuroscience Department at Maastricht University.

\section{Materials}

The experiments took place in a completely darkened room. Because some scattered light could not be avoided, a large cardboard box $(65 \times 74 \times 95 \mathrm{~cm})$ placed atop a table was used to enclose participant's field of view and shield them from stray light, thus ensuring absolute darkness (Figure 1). Head and TMS coil stability was ensured by a forehead and chinrest, together with a coil holder firmly fixing the TMS coil over the participant's skull. To allow stable fixation in the dark, dim red LED light was shone through a small hole in the back of the box, creating a fixation of $<0.25^{\circ}$ of visual angle at a viewing distance of $55 \mathrm{~cm}$ (Figure 1A). The wavelength of red light $(620-750 \mathrm{~nm})$ falls outside the response range of retinal rods (Wald, 1955) and, as such, did not interfere with dark adaptation. To ensure stable arm and hand

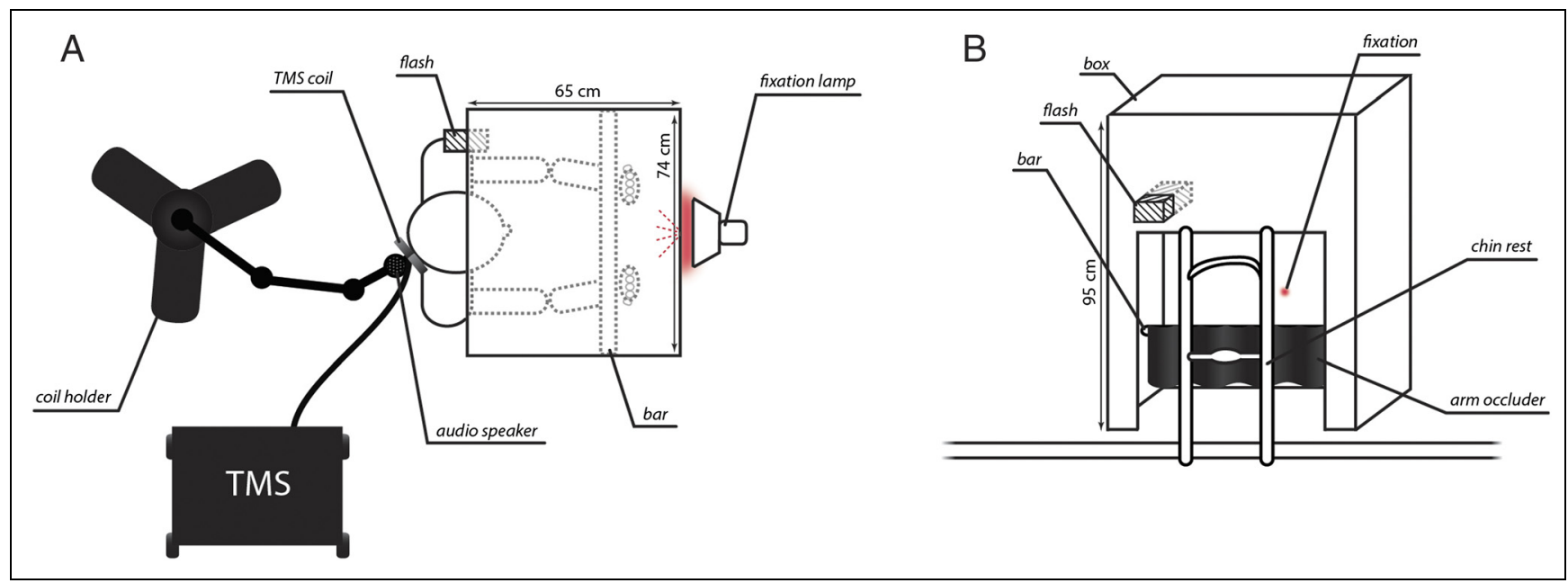

Figure 1. Experimental setup. (A) Top view of cardboard box with participant seated in the experimental setup. Stimulator and coil holder are also depicted. (B) Front view of cardboard box atop of the table, depicting also the chinrest and fixation. Images are scaled to true size. 
positions from one trial to the next, a horizontal bar was placed at $\sim 22 \mathrm{~cm}$ above the tabletop, at $42 \mathrm{~cm}$ from the front of the box. This distance required participants to raise their elbows up from the tabletop to place their hands behind the bar. Participant's arms were obscured from view by means of a black plastic arm occluder attached to the bar, and the lateral hand distance was fixed by two markers behind the bar that were readily discernable by touch. To elicit positive afterimages, a Vivitar $285 \mathrm{HW}$ Zoom Thyristor flashgun was placed in the box pointing at the white box ceiling above the participant.

Biphasic TMS was applied with a MagPro R30 stimulator (Medtronic Functional Diagnostics A/S; maximum stimulator output, $1.9 \mathrm{~T}$ ) and a figure-of-eight coil (MCB70). The coil was placed with the handle oriented laterally to the right and held throughout the experimental procedures by a custom-made coil holder, ensuring stable placement over the skull. Auditory placebo pulses were delivered over an audio speaker that was attached to the coil holder, adjacent to the TMS coil. TMS sounds, as emitted by the TMS coil, were recorded with an iRiver recording device and edited offline to filter out background noise with the Audacity software package. Volume (in $\mathrm{dB}$ ) was measured with a decibel meter for both placebo (generated by the speaker) and real (generated by the TMS coil) pulses across a range of intensities, and the volume of the placebo pulses was equated to match the volume of the real pulses. Although matched in their auditory quality, it should be noted that this placebo procedure did not elicit the same somatosensory experience as real TMS. Thus, although trials with real and placebo TMS were identical in almost all respects, differences in the somatosensory quality of the pulses were readily perceptible. To control trial timing in the dark, auditory beeps indicated the start of every new trial at 40-sec intervals. Beeps were played from a computer and were generated using the Presentation software package (Experiment 1) and MATLAB with the Psychophysics toolbox (Experiment 2; Brainard, 1997).

\section{Procedure}

Each of the two experiments consisted of two separate sessions: a preliminary session and an experimental session. The purpose of the preliminary session was (1) to check if participants could perceive phosphenes, (2) to familiarize participants with afterimages and experimental procedures, (3) to practice the rating scale, and (4) to screen participants and see whether movement of a hand resulted in perceived fading of that hand from the afterimage. This screening procedure was adapted from previous work (Rademaker, Wu, Bloem, \& Sack, 2014; Carlson, Alvarez, Wu, \& Verstraten, 2010) and required because both Experiments 1 and 2 critically depended on participant's ability to perceive fading in response to bodily movement.
During the preliminary session, participants were seated with their head in the chinrest and instructed to fixate throughout. This session consisted of 15 practice trials performed after 10 min of dark adaptation: The first three trials were to familiarize participants with afterimages, without performing a task. During the following six trials, participants placed their hands behind the horizontal bar with their palms facing toward them. After the flash, the left or right hand was removed (or both were kept stationary in Experiment 2), and participants described their percept. During the final six trials, participants practiced quantifying their subjective fading experiences, using the rating scale. For Experiment 1, we used a rating scale that relied on a relative judgment of the two hands on an 11-point scale (adapted from Hogendoorn et al., 2009), and for Experiment 2, we used a variant of this scale that allowed the hands to be judged independently on a 7-point scale (Figure 2C and D, respectively). The preliminary session took approximately $1 \mathrm{hr}$ to complete. Some participants were allowed extra practice trials in case of difficulties mastering the task and/or rating scale. Importantly, this practice allowed participants to experience the range of percepts associated with removing a hand from an afterimage and trained them to map these qualitatively rich perceptual experiences onto an internally consistent quantitative rating.

An experimental session started by positioning the coil over the right posterior part of the skull and identifying the part of EVC corresponding to the visual field position of the left hand by means of phosphene localization (Figure 2B). Once properly localized, the coil was fixed over the skull with a coil holder, and participant's phosphene thresholds were determined in an already dimmed room, using a brief staircase procedure. Subsequent stimulation was applied at $80 \%$ of phosphene threshold (mean = $31.7 \%$ of stimulator output with $S D=4.8 \%$ in Experiment 1 , and mean $=39.2 \%$ of stimulator output with $S D=10.9 \%$ in Experiment 2). After 10 min of dark adaptation, participants completed six blocks of 15 trials per block. A trial (Figure 2A) started by participants placing their hands behind the bar, and after the flash was emitted and an afterimage had formed, participants removed one of their hands (or kept both up) and after a brief ( $\sim 1 \mathrm{sec}$ ) period of observation gave a first rating. This first rating triggered the TMS or placebo pulses, after which participants again observed and rated their hands ( $\sim 1$ sec). Participants were instructed to provide ratings that were internally consistent. Participants fixated throughout each trial. To avoid drift of the afterimage relative to fixation, the red light was always switched off simultaneously with the flash, after which participants continued to fixate on the (now unlit) fixation spot. Participants could rest their arms on the tabletop in between trials.

The six experimental blocks of Experiment 1 consisted of two "hand" conditions (either the left or the right hand was removed) and three "pulse" conditions (either a 
Figure 2. Trial sequence, stimulation, and rating scales. (A) At the start of each trial, participants put both their hands up behind the black bar and started fixation aided by a small red light. When participants indicated that they were ready to start the trial, a brief flash was emitted ( $\sim 1 \mathrm{msec}$ ), and once the afterimage had developed, participants removed one of their hands (or kept both stationary). After briefly observing their hands, participants gave a first rating, which triggered placebo (auditory clicks) or TMS pulses. After the pulses, participants again briefly observed their hands and provided a second rating. The next trial started $40 \mathrm{sec}$ after the start of the previous trial, and in between trials, participants could rest their arms and move their eyes around

freely. (B) TMS was applied over the right part of EVC corresponding to the left part of visual space. To stimulate the part of the visual field containing the left hand. Before the start of the experiment phosphenes were elicited and the coil moved around until the left hand and phosphenes overlapped. During the experiment itself, stimulation was applied at $80 \%$ of phosphene threshold to ensure participants would not perceive any phosphenes. (C) Rating scale Experiment 1. A rating of zero would indicate that both hands were perceived with equal strength. A rating of -5 indicated that the left hand had faded entirely, whereas the right hand was perceived veridically. A rating of 5 meant the opposite, with the left hand being perceived as veridical and the right hand having faded completely. (D) Rating scale Experiment 2 . Half of the participants would always rate first the left and then the right hand, whereas the other half of participants always rated first the right and then the left hand.

single placebo sound pulse, a single TMS pulse, or triple TMS pulses at $10 \mathrm{~Hz}$ ). Because TMS targeted the left part of visual space overlapping with the position of the left hand, this design allowed us to evaluate the effect of EVC TMS on fading in the afterimage when the left hand was removed. Moreover, this could be compared with how TMS affected fading of the right hand and to fading during placebo instead of real TMS. For real TMS, we opted to include both single- and triple-pulse conditions because we were unsure if a single pulse below phosphene threshold would be sufficiently powerful, and more neurostimulation is elicited with three pulses. Experiment 2 consisted of three "hand" conditions (left hand was removed, right hand was removed, or both hands remained stationary) and two "pulse" conditions (triple placebo sound pulses or real triple TMS pulses at $10 \mathrm{~Hz}$ ). The stationary condition allowed us to see how TMS affected the percept of a hand when there was only gradual fading of the entire afterimage occurring naturally over time. The order of blocks was counterbalanced between participants. An experimental session lasted between 1.5 and $2 \mathrm{hr}$.

\section{RESULTS}

\section{Experiment 1}

Participants gave two ratings on a relative scale (Figure 2C)—one right before and one right after the pulse. ${ }^{1}$ If the left hand was less visible than the right, participants gave a negative rating. Conversely, positive ratings were given if the right hand was faded relative to the left. If both hands were equally visible, the rating would be " $0 . "$ Note that to equate the effects of removing the left and right hands (negative- and positive-going, respectively), we inverted the sign of the ratings given when the left hand was removed before statistical testing. Thus, one can consider the negative-going average ratings displayed in the top panel of Figure 3A as positive (and consequently, the positive-going light gray bars in Figure 3B as negative), for the purposes of interpreting all subsequent statistical analyses reported here.

To verify that participants experienced gestalt-like fading of the hand removed from the afterimage before the pulse (Figure 3A, green bars), we performed a 2 (Hand condition) $\times 3$ (Pulse condition) repeated-measures ANOVA against zero. Removing a hand from the afterimage resulted in perceived visual fading of that hand, $F(1,9)=$ 93.150, $p<.001, \eta_{\mathrm{p}}^{2}=.912$ ), with average ratings between 1.97 and 2.42 across conditions. Moreover, equal amounts of fading were experienced before the pulse, with no differences between the remove left and the remove right hand conditions, $F(1,9)=1.390, p=.269, \eta_{\mathrm{p}}^{2}=$ .134 , nor any differences between pulse conditions, $F(2$, 18) $=0.118, p=.889, \eta_{p}^{2}=.013$. Indeed, no differences were expected at this baseline rating, which was given before the application of (real or placebo) TMS pulses. 


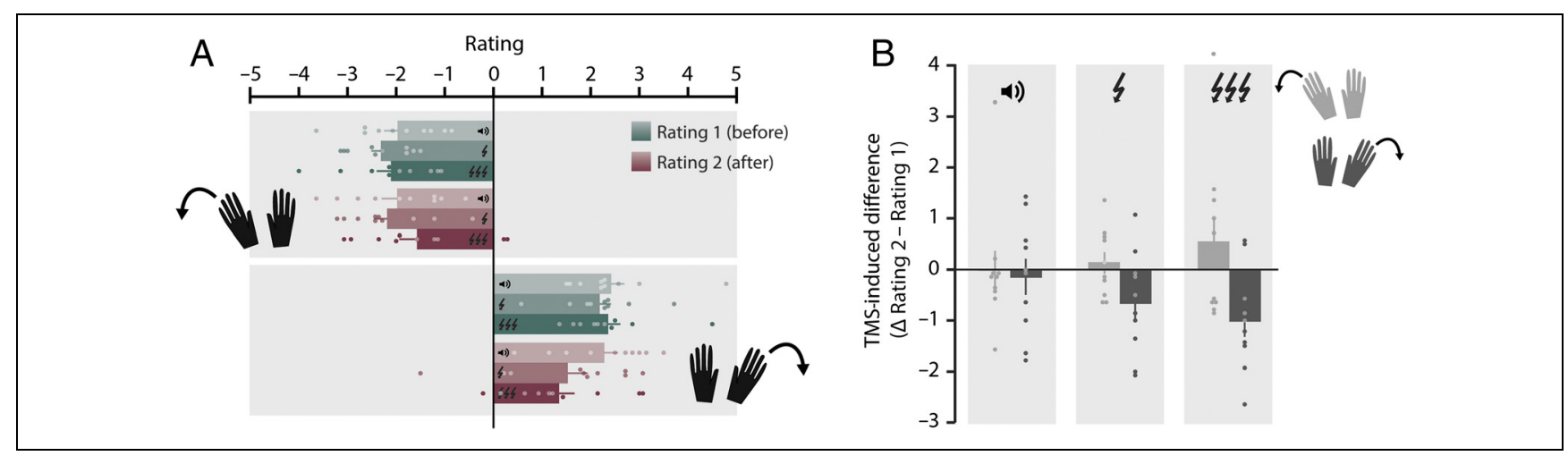

Figure 3. The effects of placebo and real TMS on fading of the left and right hands in Experiment 1. (A) Participants rate the relative visibility of their hands after removing one hand from view both before and after a pulse (placebo, single-pulse, or triple-pulse TMS). Before the pulse (green bars), there were no differences between conditions. After the pulse (red bars), there was a difference between the pulse conditions, with relative ratings closer to zero after triple-pulse TMS compared with a placebo sound pulse. This indicates that, after triple-pulse TMS, the two hands were rated as more similar in their degree of visibility. (B) Data in A are replotted to more clearly show the impact of the pulse by contrasting the ratings before and after the pulse. A difference of zero indicates no change in relative ratings before and after the pulse. For trials on which the left hand was removed (light gray), a positive score indicates that the pulse made the two hands appear more similar in terms of their visibility. Similarly, for trials on which the right hand was removed (dark gray), a negative score indicates that the pulse made the two hands appear more similar in terms of their visibility. Note that, to perform statistical testing, the scores given after left hand removal were inverted to equate the directionality of the fading effect. Dots indicate individual participant data, error bars indicate \pm 1 SEM.

Next, we investigated the impact of the pulses on perceived fading (compare green and red bars in Figure 3A). A 2 (Hand condition) $\times 3$ (Pulse condition) $\times 2$ (Before and after rating) repeated-measures ANOVA showed a significant interaction between Pulse condition and Before/after rating, $F(2,18)=3.940, p=.038, \eta_{\mathrm{p}}^{2}=$ .304 . We followed up on this interaction by first collapsing the data across the hand condition, as relative ratings were no different for the left and right hands, $F(1,9)<$ $\left.0.001, p=.997, \eta_{\mathrm{p}}^{2}<.001\right)$. Second, to compare the before and after ratings, we performed three post hoc $t$ tests, each within a given pulse condition. Perceived fading did not change between the first (before) and second (after) rating for placebo pulses (mean before $=$ $2.20, S E=0.26$ and mean after $=2.13, S E=0.28)$, $t(10)=0.214, p=.835$, nor was there a significant change with single-pulse TMS (mean before $=2.25, S E=0.19$ and mean after $=1.86, S E=0.37), t(10)=1.786, p=$ .108. When triple-pulse TMS was applied, ratings after the pulses were marginally closer to zero than before the pulses (mean before $=2.23, S E=0.26$ and mean after $=1.46, S E=0.28), t(10)=2.197, p=.056$. This indicates that the relative visibility of the two hands became more similar after triple-pulse TMS, irrespective of whether the left or right hand was removed. Third, to compare pulse conditions, we performed two repeatedmeasures ANOVAs, one within the before ratings and one within the after ratings. As already reported above, there were no differences between pulse conditions at the first (before) rating, $F(2,18)=0.118, p=.889$, $\eta_{\mathrm{p}}^{2}=.013$. However, at the second (after) rating, there was a significant main effect of Pulse condition, $F(2,18)=$ $6.221, p=.009, \eta_{\mathrm{p}}^{2}=.409$, driven by the difference between placebo and triple-pulse TMS, $t(9)=3.02$, $p=.043$, Bonferroni-corrected.
Figure $3 \mathrm{~B}$ replots the data in Figure $3 \mathrm{~A}$ by showing the difference between the before and after ratings. Here, the further the difference score is away from zero, the more similar the hands became after the pulse (in positive and negative directions for removing the left and right hands, respectively). An analysis of the difference scores in a 2 (Hand condition) $\times 3$ (Pulse condition) repeated-measures ANOVA revealed a significant main effect of Pulse condition, $F(2,18)=3.960, p=.038$, $\eta_{\mathrm{p}}^{2}=.306$, but no effect of Hand condition, $F(1,9)=$ $1.338, p=.277, \eta_{\mathrm{p}}^{2}=.129$, and no interaction, $F(2,18)=$ $0.827, p=.453, \eta_{\mathrm{p}}^{2}=.084$. The main effect of Pulse condition was driven by a trending difference between placebo and triple-pulse TMS, $t(9)=2.816, p=.06$, Bonferroni-corrected, indicating the two hands were perceived as more similar after a triple TMS pulse, compared with placebo. Together, these results suggest that triple-pulse TMS significantly reduced the amount of fading participants experienced after removing their hand from an afterimage, irrespective of the hand (the left or right) that was removed.

\section{Experiment 2}

The relative rating scale employed in Experiment 1 comes with two important limitations. First, under the assumption that TMS over the right EVC exclusively targets the location of visual space encompassing the left hand, the relative rating should reflect perceived changes exclusively at this location. Thus, the TMS-induced reduction in relative ratings when the left hand was removed implies that TMS strengthened the visual representation of the left hand, making it more similar to the stationary right hand. Following the same logic, the TMS-induced reduction in the relative rating when the right hand 
was removed implies that TMS weakened the quality of the percept of the stationary left hand, making it more similar in quality to the faded right hand. However, we cannot exclude alternative explanations for the results in Experiment 1. For example, if TMS over the right EVC were able to also affect perception of the right hand, relative ratings would not dissociate between what is happening at the two hands. To illustrate this point, consider a trial during which the left hand was removed: If the relative rating was closer to zero after applying TMS, this could mean that the perceived quality of the left hand was improved or, alternatively, that the quality of the right hand was reduced. Both would make the hands appear qualitatively more similar. A second limitation of the relative rating scale is that it is not sensitive to detecting overall fading of the hands in the afterimage that occurs naturally over time: Two hands that were both very crisp would receive a rating of zero, whereas two hands that had nearly faded entirely, but to an equal extent, would also get a rating of zero.

To address these limitations, Experiment 2 required participants to rate the left and right hands independently, and on an absolute scale from 0 to 6 (where 0 indicated complete fading of the hand and 6 indicated the crispest possible afterimage of the hand). This allowed us to directly observe the effect of TMS on each individual hand and also to track overall fading levels of the hands in the afterimage. Furthermore, Experiment 2 included a condition in which neither hand was removed, allowing us to observe potential effects of TMS on an afterimage in the absence of gestalt-like fading. Only triple placebo sound pulses and real triple TMS pulses were used in Experiment 2.

We first analyzed the data as we did in Experiment 1 to verify that our effects replicated. To this end, a relative rating was calculated by subtracting the ratings of the individual hands (left minus right; Figure 4). As before, we inverted the sign of the relative ratings for the left hand removal condition before statistical testing to equate the directionality of the fading effect between the two hands. We confirmed that removing both the left, $F(1,9)=$ $13.740, p=.005, \eta_{\mathrm{p}}^{2}=.604$, and right, $F(1,9)=$ 20.896, $p=.001, \eta_{\mathrm{p}}^{2}=.699$, hand from the afterimage resulted in gestalt-like fading before the pulses were applied (Figure 4A, green bars; repeated-measures ANOVAs against zero), and the extent of this fading was comparable between removing the left and right hands, $F(1,9)=$ $0.510, p=.493, \eta_{\mathrm{p}}^{2}=.054$. When both hands were kept up, the right hand was relatively less visible than the left before the pulses, $F(1,9)=7.781, p=.021, \eta_{\mathrm{p}}^{2}=.464$, as indicated by the positive-going relative difference. However, the relative ratings were only slightly above zero ( 0.17 and 0.22 for placebo and real TMS, respectively), and these results did not hold up in direct $t$ tests against zero: $t(9)=1.98, p=.079$ and $t(9)=1.75, p=.11$ for placebo and real TMS, respectively.

We again assessed the differences between before and after ratings (Figure 4B). A 3 (Hand condition) $\times 2$ (Pulse condition) repeated-measures ANOVA showed a main effect of Hand condition, $F(2,18)=5.791, p=.011, \eta_{\mathrm{p}}^{2}=$ .392 , driven by a marginal difference between the both hands up and remove right conditions, $t(9)=-2.845$, $p=.058$, but not by any differences between the remove left and remove right conditions, $t(9)=1.383, p=.600$. Importantly, ratings of the two hands became more similar after real TMS pulses than after placebo pulses, $F(1,9)=$ 8.077, $p=.019, \eta_{\mathrm{p}}^{2}=.473$. Specifically, a larger deviation away from zero on the $y$ axis for real TMS indicates that the two hands went from relatively different at rating 1 (before TMS) to relatively similar at rating 2 (after
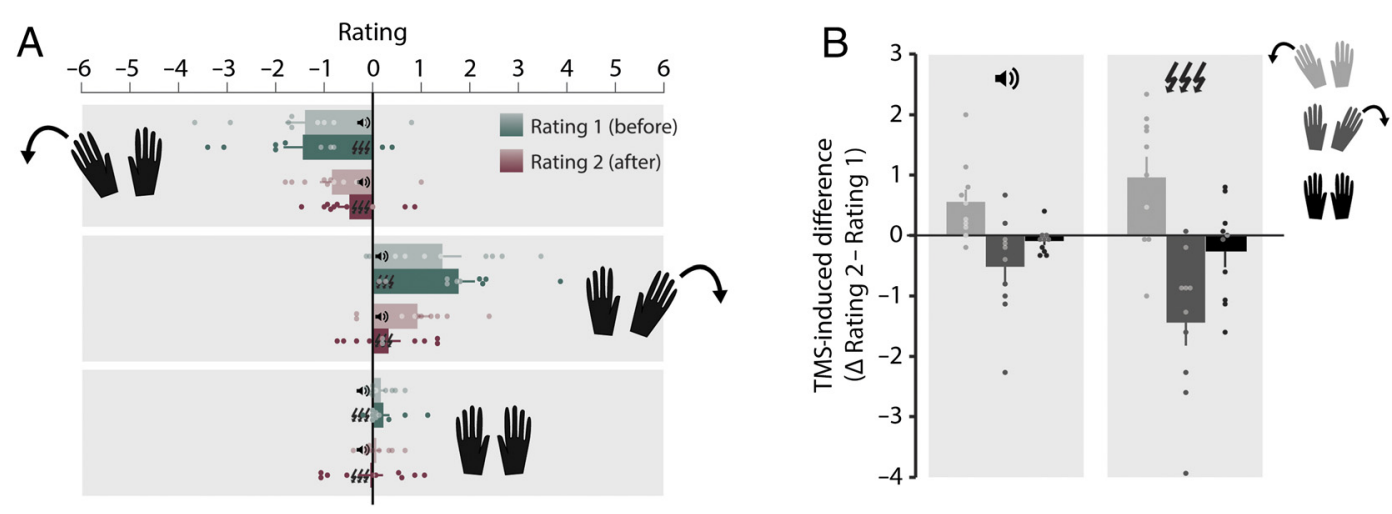

Figure 4. The effects of placebo and real TMS on fading of the left and right hands in Experiment 2. (A) Relative ratings of fading calculated from the absolute ratings participants had provided for individual hands. Note that the range of possible ratings in Experiment 2 (from -6 to 6 ) is slightly different from that in Experiment 1 (from -5 to 5 ). To ensure that both experiments had an uneven number of rating options (with zero included), an 11-point scale was used in Experiment 1 and a 7-point absolute scale (i.e., 0-6, resulting in a 13-point relative scale) was used in Experiment 2 . The effect of TMS pulses on fading was evaluated by calculating the difference between ratings (after minus before), as is shown in B. A positive deflection along the $y$ axis indicates that ratings became more positive after TMS, as was the case when the left hand was removed (i.e., negative relative ratings before TMS would tend toward zero after TMS). Conversely, when the right hand was removed, a negative deflection along the $y$ axis indicates that ratings became more positive after TMS. Thus, the two hands were perceived as more similar after real TMS, irrespective of the hand that was removed from the afterimage. Dots indicate individual participant data, error bars indicate \pm 1 SEM. 
TMS). There was no significant interaction, $F(2,18)=$ 3.164, $p=.066, \eta_{\mathrm{p}}^{2}=.260$. These results replicate the findings of Experiment 1, insofar that real TMS caused perceived relative differences between a removed and a stationary hand to decrease compared with placebo.

Next, we analyzed the data from Experiment 2 by capitalizing on the absolute rating scale. Beyond what can be gleaned from relative difference scores, absolute ratings allowed us to assess fading at each hand individually. We first calculated the difference between the before and after ratings (Figure 5). A difference score of zero indicated no change in the perceived quality of the hand, whereas negative scores indicated an increase in the amount of fading at the second (after) compared with the first (before) rating. Note that in Figure 5 all scores are negative, indicating overall fading over time between the first and second rating.

For trials on which one of the hands was removed, we organized these difference scores according to the action at the rated hand. Specifically, we grouped difference scores by the rated hand being "removed" (i.e., right hand ratings when the right hand was removed and left hand ratings when the left hand was removed) or "stationary" (i.e., right hand ratings when the left hand was removed and left hand ratings when the right hand was removed). On trials where both hands were kept up, ratings of the left and right hands both refer to a situation in which a "stationary" hand was rated.

The results of a 3 (Hand condition) $\times 2$ (Hand rated) $\times$ 2 (Pulse condition) repeated-measures ANOVA showed

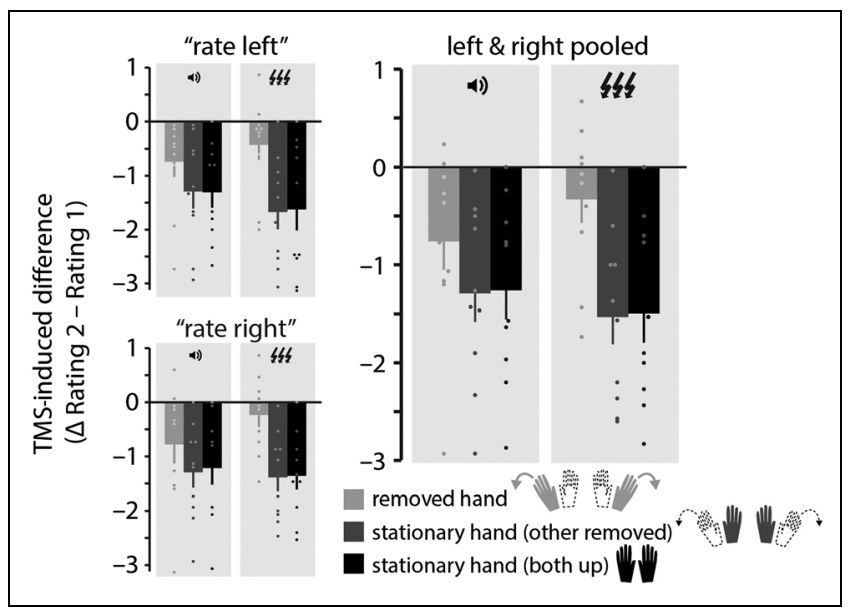

Figure 5. The effects of placebo and real TMS on fading of the left and right hands in Experiment 2. (A) Difference scores (rating after rating before) are plotted against what occurred at the rated hand (i.e., three hand conditions). Hand condition was organized in three event types: (1) the rated hand was removed, (2) the rated hand was stationary and the only hand kept up, and (3) the rated hand was stationary while both hands were kept up. More negative going scores indicate that the rated hand became less visible over time (i.e., between the first and second rating). (B) Replots the data in A pooled across the left and right hands. Dots indicate individual participant data, error bars indicate \pm 1 SEM. no main effect of the hand being rated, $F(1,9)=$ $2.002, p=.191, \eta_{\mathrm{p}}^{2}=.182$ (Figure $5 \mathrm{~A}$ ), suggesting that any changes in response to the experimental manipulation occurred similarly in both the left and the right hand. Importantly, there was a significant interaction between Hand condition and Pulse condition, $F(1,9)=11.265$, $p=.001, \eta_{\mathrm{p}}^{2}=.556$.

To explore this interaction, we first collapsed the data across the hand being rated (left or right). Next, we wanted to assess fading at the removed and stationary hands and, critically, how TMS influenced fading in the different hand removal conditions. To this end, we ran two 3-way repeated-measures ANOVA, one for each pulse condition. Both ANOVAs revealed differences in fading between the three hand conditions (placebo TMS: $F(2$, $18)=4.766, p=.022, \eta_{\mathrm{p}}^{2}=.346$, and real TMS: $F(2$, 18) $\left.=12.173, p<.001, \eta_{\mathrm{p}}^{2}=.575\right)$. However, for placebo pulses, none of the post hoc pairwise comparisons remained significant (all $p s>.12$ ), indicating that the differences in fading were not robust between the three hand conditions. Note also that in the removed hand condition, there exists a ceiling effect: A removed and already faded hand has little room to fade further. Thus, the amount of fading that naturally occurs over time for a stationary hand is likely larger than the amount of fading still possible for a removed hand, also in the placebo condition. In contrast, post hoc tests for real TMS showed significant differences between ratings of the removed hand and the stationary hand, both when the stationary hand was the only hand up, $t(9)=3.566$, $p=.018$, Bonferroni-corrected, as well as when both hands were up, $t(9)=3.566, p=.018$, Bonferronicorrected.

Next, we assessed how TMS condition influenced fading within the separate hand conditions, which was tested with three 2-way ANOVAs, one for each hand condition. When the hand was removed, placebo and real TMS had a different impact on perceived fading, $F(1,9)=5.417$, $p=.045, \eta_{\mathrm{p}}^{2}=.376$, with scores more proximal to zero for real (mean $=-0.33, S E=0.24)$ compared with placebo pulses (mean $=-0.76, S E=0.29$ ). Because negative scores indicate that the hand faded from the first (before) to the second (after) rating, the real TMS score being more proximal to zero compared with placebo pulses suggests that real TMS reduced the amount of fading observed for the removed hand.

For stationary hands, real TMS caused marginally (but not significantly) more fading compared with placebo TMS, both when the stationary hand was the only hand up, $F(1,9)=4.606, p=.060, \eta_{\mathrm{p}}^{2}=.339$, and when both hands were up, $F(1,9)=4.046, p=.075, \eta_{\mathrm{p}}^{2}=.310$. In terms of effect sizes, real TMS scores were on average $-1.53(S E=0.28)$ and $-1.49(S E=0.30)$ for one and both hands up, respectively. Scores with placebo TMS were $-1.29(S E=0.29)$ and $-1.26(S E=0.29)$ for one and both hands up, respectively. Although not statistically significant, the idea that fading ensues when TMS 
is used to disrupt processing of a visual stimulus (here: a stationary hand) is in line with previous work showing fading after TMS over intraparietal sulcus (Kanai et al., 2008).

\section{DISCUSSION}

Activity in EVC correlates with subjective percepts, even when a percept is illusory and not a direct reflection of retinal inputs (Sperandio et al., 2012; Murray et al., 2006; Meng et al., 2005; Sasaki \& Watanabe, 2004). Such findings imply that visual fading occurs when bottom-up sensory information is muted, either via local inhibition or by targeted top-down inhibition. The role of top-down influences is supported by the fact that fading occurs in gestalt-like chunks (Billock \& Tsou, 2004), hinting at the involvement of cortical areas with more complex response profiles. The experiments presented here set out to explore the mechanisms behind visual fading in positive afterimages, induced through a multisensory conflict between vision and proprioception. Using gestalt-like fading of a hand removed from a retinally stabilized afterimage, we showed that online stimulation of EVC partially reversed visual fading.

In Experiment 1, participants removed either their left or right hand from a positive afterimage, after which the removed hand faded from view. Subsequently, participants rated the relative intensity of their two hands twice-directly before and directly after single, triple, or placebo TMS. TMS was applied over the right EVC location corresponding to the visual field location occupied by the left hand. After triple-pulse TMS, the two hands were rated as more similar, compared with after placebo or single-pulse TMS. Curiously, the effect of triple-pulse TMS over the right EVC was not specific to the condition in which the left hand was removed (and faded) - the two hands were also rated as more similar after removal (and fading) of the right hand positioned contralateral to the part of visual space targeted by TMS. Thus, triplepulse TMS impacted perception both when a hand contralateral to the targeted hemisphere was removed and when a hand ipsilateral to the targeted hemisphere was removed. Notably, the rating scale used in Experiment 1 was based on the relative clarity of the two hands in the afterimage, obscuring the possible reason underlying this bilaterality. Specifically, when the right hand was removed and the two hands were rated as more similar after triple-pulse TMS, this could be because (1) an increase in the perceptual quality of the removed right hand (an effect ipsilateral to the TMS pulses), (2) a reduction of the perceptual quality of the stationary left hand (an effect contralateral to the TMS pulses), or (3) a combination of both.

In Experiment 2, participants removed their left or right hand from the afterimage or kept both hands up. Participants then rated the absolute intensity of their two hands separately, both before and after three placebo or real TMS pulses. First, in an analysis aimed at replicating the relative ratings provided in Experiment 1, the application of triple-pulse TMS resulted in ratings of the hands becoming more similar (compared with placebo pulses), indeed replicating our findings from Experiment 1. Second, the absolute ratings of the individual hands further allowed us to evaluate the source of this effect. We found that triple-pulse TMS resulted in a relative strengthening of the percept of both the left and right hands. These findings imply that TMS over the right EVC can help reverse the effects of visual fading in positive afterimages and that the impact of the pulses is not hemifield specific.

Why would triple-pulse TMS over the right EVC, aimed at the visuospatial location of the left hand, reduce fading at both the left and right hands? First, TMS could have spread to the contralateral hemisphere through monosynaptic transcollosal connections (Berlucchi \& Rizzolatti, 1968). Indeed, previous work combining EEG with TMS over occipital cortex has demonstrated that a single TMS pulse to one hemisphere can spread to the contralateral hemisphere within $28 \mathrm{msec}$ after stimulation (Ilmoniemi et al., 1997). Given that we applied multiple pulses and studied an effect that evolves on a timescale of seconds, rather than milliseconds, it is possible that the observed bilateral effects were due to interhemispheric spreading of TMS pulses.

Second, the effect of TMS over EVC might propagate to upstream areas with large visual receptive fields that can encompass the spatial location of both hands. If such areas in turn provide feedback to EVC that results in visual fading, their ability to do so-to either of the removed hands-could be disrupted. In our paradigm, visual fading of a hand arises from a multimodal conflict between vision and proprioception. A putative mechanism for modulation of EVC activity through other modalities is via back-projections originating in multimodal integration areas, such as posterior parietal cortex (PPC; Bolognini \& Maravita, 2007; Macaluso et al., 2000). Anatomically, early visual areas are indeed innervated by connections originating in multimodal areas of the brain (Lewis \& Van Essen, 2000; Blatt, Andersen, \& Stoner, 1990; Cavada \& GoldmanRakic, 1989).

The PPC plays an important role in multimodal integration (Maravita, Spence, \& Driver, 2003). In monkeys, the PPC is a known integration site of somatosensory and visuospatial information (Hihara et al., 2006; Graziano, Cooke, \& Taylor, 2000; Iwamura, 1998; Iwamura, Iriki, \& Tanaka, 1994; Sakata, Takaoka, Kawarasaki, \& Shibutani, 1973), with visually responsive neurons in ventral premotor cortex of macaque that can continually update their visual receptive fields to track an effector moving through space (Graziano, Hu, \& Gross, 1997). Recent neuroimaging work in humans has implicated similar loci of visual-proprioceptive integration (Limanowski \& Blankenburg, 2016). When participants saw a photorealistic virtual arm at a location that was congruent with 
the position of their real arm, increased activity was observed in PPC, compared with when the virtual and real arm were at incongruent locations. Interestingly, when visual and proprioceptive information were congruent, correlations between integration areas, such as PPC, and V1 implied direct communication between these areas (Limanowski \& Blankenburg, 2016). A follow-up study investigated hand laterality by looking at congruent and incongruent presentations of both the left and right hands positioned in their respective hemifields. A cluster in left inferior parietal lobule responded preferentially to congruent visuoproprioceptive hand information for both the left and right hands, even with the hands presented in different (the left and right) hemifields (Limanowski \& Blankenburg, 2017). These studies highlight cortical sites, like the PPC, at which proprioceptive information coexist with visual representations of both hands.

Importantly, multimodal interactions have the capacity to alter EVC activity, as has been demonstrated by studies on cross-modal facilitation effects. For example, when tactile stimulation of the hand coincided with a visual stimulus on the same side of space, detection of the visual stimulus was facilitated, and visual cortex activity was enhanced (Macaluso et al., 2000). Similarly, several studies have assessed visual cortex excitability by inducing visual phosphenes, which are briefly perceived flashes of light in response to a TMS pulse over EVC. A higher level of cortical excitability is assumed to lower the threshold for eliciting a phosphene (de Graaf, Duecker, Stankevich, Ten Oever, \& Sack, 2017). Visual cortex excitability was increased at locations of the visual field that coincided with the location at which a hand was touched (i.e., "coincident locations"; Ramos-Estebanez et al., 2007). This effect occurred both with the hands crossed and uncrossed, always showing increased excitability when phosphene location and touch were coincident, irrespective of the hand that was touched (Bolognini \& Maravita, 2007). After repetitive $1 \mathrm{~Hz}$ TMS of PPC (but not EVC), crossmodal facilitation of EVC still occurred at the coincident location when the hands were uncrossed, but when the hands were crossed, it was the noncoincident location that led to increased EVC excitability (i.e., when the tactile stimulus occurred at a location in space corresponding to the ipsilateral hemisphere).

Together, these results suggest that PPC is responsible for binding visual and proprioceptive inputs into a common reference frame that relates to the body and that top-down influences from parietal multimodal integration areas can directly innervate and impact processing in EVC. In the context of the current experiments, if integrated feedback is sent back to EVC, the PPC could indeed provide a reference frame that incorporates information from both hands (represented in both hemispheres).

In our paradigm, a sensory conflict arises when a hand is removed from the afterimage, resulting in perceptual fading of the removed hand. We propose that such visual fading results from an attempt to match the visual percept to the proprioceptive experience. Although this type of fading (through sensory conflict) is likely instantiated via feedback from areas integrating multisensory information (like PPC), continued perceptual fading could be implemented at the level of EVC itself. In either case, visual signals might be actively inhibited in EVC, which would result in perceptual visual fading of the hand. In turn, TMS over EVC could release this inhibition, thereby increasing the perceptual quality of a faded hand. Indeed, TMS has been shown to have the potential to release cortical inhibition in EVC (Ling, Pearson, \& Blake, 2009).

In this context, it is important to note that fading of the afterimage as a whole also occurs in the absence of any sensory conflict, and removing a hand could simply be considered a way to speed up the fading process at a punctate visual location. Future work could address whether fading and possible inhibitory processes in EVC are sustained through continued top-down feedback signals or via local processes. Such work could focus on the role of PPC in addition to the role of EVC. If PPC is indeed the locus of the original inhibitory signal to EVC, TMS at various time points during the perceptual fading paradigm (e.g., before, during, of after removing the hand) might differentially impact the perception of the afterimage.

As a first cautionary note, we would like the reader to consider that the general mechanisms behind visual fading as a result from proprioceptive feedback, such as those observed in the positive images paradigm, are not entirely understood. Therefore, the suggested inhibition at the level of EVC that we consider a driving force behind visual fading in positive afterimages may or may not generalize as a catch-all mechanism that applies to the visual fading literature more broadly.

As a second cautionary note, we want to point out that real and placebo pulses were matched in almost every way but that they did not match in their somatosensory quality. The experiments reported here were completed in total darkness, which prevented us from randomly interleaving blocks of real and placebo trials using more conventional sham procedures (such as intermittently switching to a sham coil or moving the TMS coil to vertex). Nevertheless, the single-pulse condition in Experiment 1 served as a more active control, because it did have a tactile sensation associated with it. Single pulses did not elicit significant differences in ratings before and after the pulse. It is reasonable to assume that fewer pulses lead to less overall neural firing compared with more pulses; thus, we are confident that our effects are a function of TMS and not the presence or absence of a tactile sensation. Furthermore, if pulse condition had somehow systematically altered the way participants rated their afterimages from one block to the next, we would expect to see differences between conditions in participant's prepulse ratings — which we did not. Thus, we do not think that the mere somatosensation of a pulse can explain the current findings. 
Transients in sensory inputs are generally required for conscious perception, whereas stable inputs (such as retinally stabilized afterimages) are filtered out of the perceptual experience. Here, we demonstrate that TMS over EVC can make a hand that has faded from a positive afterimage more visible again, signifying a causal role for EVC processes in perceptual fading.

\section{Acknowledgments}

This work was supported by the European Union's Horizon 2020 Research and Innovation Program under the Marie Sklodowska-Curie Grant agreement no. 743941 to R. L. R. This work is part of the VICI Research Program No. 453-15008 awarded to A. T. S., (partly) financed by the Netherlands Organization for Scientific Research (NWO).

Reprint requests should be sent to Rosanne L. Rademaker, Department of Psychology, UC San Diego, 9500 Gilman drive, La Jolla, CA 92093, or via e-mail: rosanne.rademaker@gmail.com.

\section{Note}

1. Data availability statement: All data collected during the experiments presented here are publicly available through the Open Science Framework at https://osf.io/bnzm8/.

\section{REFERENCES}

Berlucchi, G., \& Rizzolatti, G. (1968). Binocularly driven neurons in visual cortex of split-chiasm cats. Science, 159, 308-310.

Billock, V. A., \& Tsou, B. H. (2004). What do catastrophic visual binding failures look like? Trends in Neurosciences, 27, 84-89.

Blatt, G. J., Andersen, R. A., \& Stoner, G. R. (1990). Visual receptive field organization and cortico-cortical connections of the lateral intraparietal area (area LIP) in the macaque. Journal of Comparative Neurology, 299, 421-445.

Bolognini, N., \& Maravita, A. (2007). Proprioceptive alignment of visual and somatosensory maps in the posterior parietal cortex. Current Biology, 17, 1890-1895.

Brainard, D. H. (1997). The Psychophysics Toolbox. Spatial Vision, 10, 433-436.

Bross, M. (2000). Emmert's law in the dark: Active and passive proprioceptive effects on positive visual afterimages. Perception, 29, 1385-1391.

Carey, D. P., \& Allan, K. (1996). A motor signal and "visual" size perception. Experimental Brain Research, 110, 482-486.

Carlson, T. A., Alvarez, G., Wu, D.-A., \& Verstraten, F. A. J. (2010). Rapid assimilation of external objects into the body schema. Psychological Science, 21, 1000-1005.

Cavada, C., \& Goldman-Rakic, P. S. (1989). Posterior parietal cortex in rhesus monkey: I. Parcellation of areas based on distinctive limbic and sensory corticocortical connections. Journal of Comparative Neurology, 287, 393-421.

Darwin, E. (1794). Zoonomia; or the laws of organic life (4th American ed.). Philadelphia, PA: Edward Earle.

Davies, P. (1973). Effects of movements upon the appearance and duration of a prolonged visual afterimage: 1 . Changes arising from the movement of a portion of the body incorporated in the afterimaged scene. Perception, 2, 147-153.

de Graaf, T. A., Duecker, F., Stankevich, Y., Ten Oever, S., \& Sack, A. T. (2017). Seeing in the dark: Phosphene thresholds with eyes open versus closed in the absence of visual inputs. Brain Stimulation, 10, 828-835.

Ditchburn, R. W., \& Ginsborg, B. L. (1952). Vision with a stabilized retinal image. Nature, 170, 36-37.

Graziano, M. S. A., Cooke, D. F., \& Taylor, C. S. R. (2000). Coding the location of the arm by sight. Science, 290 1782-1786.

Graziano, M. S. A., Hu, X. T., \& Gross, C. G. (1997). Visuospatial properties of ventral premotor cortex. Journal of Neurophysiology, 77, 2268-2292.

Gregory, R. L., Wallace, J. G., \& Campbell, F. W. (1959). Changes in the size and shape of visual after-images observed in complete darkness during changes of position in space. Quarterly Journal of Experimental Psychology, $11,54-55$

Hay, J. C., Pick, H. L., \& Ikeda, K. (1965). Visual capture produced by prism spectacles. Psychonomic Science, 2, 215-216.

Hihara, S., Notoya, T., Tanaka, M., Ichinose, S., Ojima, H., Obayashi, S., et al. (2006). Extension of corticocortical afferents into the anterior bank of the intraparietal sulcus by tool-use training in adult monkeys. Neuropsychologia, 44, 2636-2646.

Hogendoorn, H., Kammers, M. P. M., Carlson, T. A., \& Verstraten, F. A. J. (2009). Being in the dark about your hand: Resolution of visuo-proprioceptive conflict by disowning visible limbs. Neuropsychologia , 47, 2698-2703.

Ilmoniemi, R. J., Virtanen, J., Ruohonen, J., Karhu, J., Aronen, H. J., Näätänen, R., et al. (1997). Neuronal responses to magnetic stimulation reveal cortical reactivity and connectivity. NeuroReport, 8, 3537-3540.

Iwamura, Y. (1998). Hierarchical somatosensory processing. Current Opinion in Neurobiology, 8, 522-528.

Iwamura, Y., Iriki, A., \& Tanaka, M. (1994). Bilateral hand representation in the postcentral somatosensory cortex. Nature, 369, 554-556.

Kanai, R., Muggleton, N. G., \& Walsh, V. (2008). TMS over the intraparietal sulcus induces perceptual fading. Journal of Neurophysiology, 100, 3343-3350.

Komatsu, H. (2006). The neural mechanisms of perceptual filling-in. Nature Reviews Neuroscience, 7, 220-231.

Lewis, J. W., \& Van Essen, D. C. (2000). Corticocortical connections of visual, sensorimotor, and multimodal processing areas in the parietal lobe of the macaque monkey. Journal of Comparative Neurology, 428, 112-137.

Limanowski, J., \& Blankenburg, F. (2016). Integration of visual and proprioceptive limb position information in human posterior parietal, premotor, and extrastriate cortex. Journal of Neuroscience, 36, 2582-2589.

Limanowski, J., \& Blankenburg, F. (2017). Posterior parietal cortex evaluates visuoproprioceptive congruence based on brief visual information. Scientific Reports, 7, 16659.

Ling, S. T., Pearson, J., \& Blake, R. (2009). Dissociation of neural mechanisms underlying orientation processing in humans. Current Biology, 19, 1458-1462.

Macaluso, E., Frith, C. D., \& Driver, J. (2000). Modulation of human visual cortex by crossmodal spatial attention. Science, 289, 1206-1208.

Maravita, A., Spence, C., \& Driver, J. (2003). Multisensory integration and the body schema: Close to hand and within reach. Current Biology, 13, R531-R539.

Martinez-Conde, S., Macknik, S. L., \& Hubel, D. H. (2004). The role of fixational eye movements in visual perception. Nature Reviews Neuroscience, 5, 229-240.

Martinez-Conde, S., Otero-Millan, J., \& Macknik, S. L. (2013). The impact of microsaccades on vision: Towards a unified theory of saccadic function. Nature Reviews Neuroscience, 14, 83-96.

Mendola, J. D., Conner, I. P., Sharma, S., Bahekar, A., \& Lemieux, S. (2006). fMRI measures of perceptual filling-in in 
the human visual cortex. Journal of Cognitive Neuroscience, $18,363-375$.

Meng, M., Remus, D. A., \& Tong, F. (2005). Filling-in of visual phantoms in the human brain. Nature Neuroscience, 8 , $1248-1254$.

Murray, S. O., Boyaci, H., \& Kersten, D. (2006). The representation of perceived angular size in human primary visual cortex. Nature Neuroscience, 9, 429-434.

Rademaker, R. L., Wu, D.-A., Bloem, I. M., \& Sack, A. T. (2014). Intensive tool-practice and skillfulness facilitate the extension of body representations in humans. Neuropsychologia, 56, 196-203.

Ramos-Estebanez, C., Merabet, L. B., Machii, K., Fregni, F., Thut, G., Wagner, T. A., et al. (2007). Visual phosphene perception modulated by subthreshold crossmodal sensory stimulation. Journal of Neuroscience, 27, 4178-4181.

Riggs, L. A., Ratliff, F., Cornsweet, J. C., \& Cornsweet, T. N. (1953). The disappearance of steadily fixated visual test objects. Journal of the Optical Society of America, 43, 495-501.

Rossi, S., Hallett, M., Rossini, P. M., Pascual-Leone, A., \& The Safety of TMS Concensus Group. (2009). Safety, ethical considerations, and application guidelines for the use of transcranial magnetic stimulation in clinical practice and research. Clinical Neurophysiology, 120, 2008-2039.

Sakata, H., Takaoka, Y., Kawarasaki, A., \& Shibutani, H. (1973). Somatosensory properties of neurons in the superior parietal cortex (area 5) of the rhesus monkey. Brain Research, 64, 85-102.

Sasaki, Y., \& Watanabe, T. (2004). The primary visual cortex fills in color. Proceedings of the National Academy of Sciences, U.S.A., 101, 18251-18256.

Shimojo, S., Kamitani, Y., \& Nishida, S. (2001). Afterimage of perceptually filled-in surface. Science, 293, 1677-1680.

Sperandio, I., Chouinard, P. A., \& Goodale, M. A. (2012). Retinotopic activity in $\mathrm{V} 1$ reflects the perceived and not the retinal size of an afterimage. Nature Neuroscience, 15, 540-542.

Troxler, D. I. P. V. (1804). Über das Verschwinden gegebener Gegenstände innerhalb unseres Gesichtskreises. Ophthalmologische Bibliothek, 2, 1-53.

Wald, G. (1955). The photoreceptor process in vision. American Journal of Ophthalmology, 40, 18-41. 